\title{
Investigação de variável adequada ao controle automático de vazão sem uso de sensores em campos irrigados por meio de análise multivariada
}

\author{
Investigation of variable appropriately to the automatic flow control without the use of sensors in \\ fields irrigated by means of multivariate analysis
}

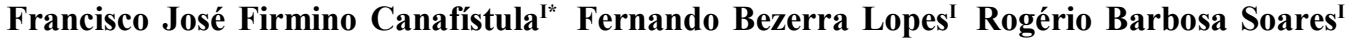 \\ Eunice Maia de Andrade ${ }^{\text {II }}$ Adunias dos Santos Teixeira ${ }^{\text {II }}$
}

RESUMO

\begin{abstract}
Utilizar inversores no controle de vazão de sistemas de bombeamento por meio da variação de sua velocidade pode ter uma maior viabilidade, caso o custo de sensores seja minimizado. Este trabalho investiga, por intermédio de análise multivariada, uma variável adequada ao pseudocontrole da vazão de um sistema de irrigação que economize o uso de sensores e simplifique o sistema de controle. Uma bancada de ensaio para coleta dados foi montada no laboratório e os dados foram processados no Statistical Package for the Social Sciences (SPSS). A análise de agrupamento hierárquico utilizando o método de Ward com base na distância euclidiana quadrática mostrou um grupo composto de quatro variáveis, estando presente nesse grupo a vazão, voltas no registro, altura do nivel de água da calha parshall e a corrente elétrica, indicativo de que a corrente elétrica pode ser usada para o pseudocontrole, pois o inversor mede essa variável. A análise fatorial também indicou uma forte correlação da corrente elétrica com a vazão do sistema. A pressão foi o parâmetro da primeira componente da análise fatorial que apresentou a maior correlação com a vazão, no entanto o uso dessa variável para o controle automático demanda o uso de sensores.
\end{abstract}

Palavras-chave: agricultura de precisão, instrumentação, sensores.

\section{ABSTRACT}

Using inverters to control flow of pumping systems by means of changes in speed may be more viable if the cost of sensors is minimized. This work investigates through multivariate analysis an adequate variable for the pseudo control of the flow of an irrigation system that saves the use of sensors and simplifies the control system. A bench test to collect data was mounted in the laboratory and the data were processed using
Statistical Package for the Social Sciences (SPSS). The hierarchical cluster analysis using the Ward method based on the squared Euclidean distance showed a group composed of four variables, being present in that group flow, turns on the register, water level of the Parshall weir and electric current, indicating that the electric current can be used for pseudo control because the inverter measures this variable. The factor analysis also indicated a strong correlation between the electric current and the system flow. In the first Principal Component, the pressure showed the highest correlation with the flow, although the use of this variable for automated control requires the use of sensors.

Key words: precision agriculture, instrumentation, sensors.

\section{INTRODUÇÃO}

Poucos são os ramos do conhecimento e as atividades humanas que podem dispensar o apoio de técnicas estatísticas em seu desenvolvimento. Uma analise de sensibilidade feita nos fenômenos que nos cercam nos remete à conclusão de que tais técnicas estão participando cada vez mais do nosso cotidiano. Essa tendência torna-se mais acentuada à medida que se expandem os recursos oferecidos pela informática, já que eles facilitam sobremaneira a análise dos dados (CORRAR et al., 2007). O desenvolvimento da agricultura de precisão está associado a técnicas de irrigação que tem sua eficiência e complexidade modificadas devido a muitas variáveis que atuam no sistema. Aplicar estatística por intermédio de

IPrograma de Pós-graduação em Engenharia Agrícola, Departamento de Engenharia Agrícola, Universidade Federal do Ceará (UFC), Av. Mister Hull, s/n, Bloco 804, CP 12168, 60455-970, Fortaleza, CE, Brasil. E-mail: firmino@ufc.br. Autor para correspondência. "Departamento de Engenharia Agrícola, UFC, Fortaleza, CE, Brasil. 
ferramentas computacionais, na busca das variáveis que tenham uma maior representatividade na eficiência dos sistemas de irrigação, pode trazer menor complexidade no controle da planta.

Nos modernos processos de produção o controle automático é importante e produz meios para melhorar os sistemas dinâmicos que integram esses processos (OGATA, 2003). Alguns desses métodos são complexos e a elaboração do seu projeto demanda experiência. Muitas vezes, na implementação do projeto de controle, além da necessidade de experiência técnica nessa fase (WAHAB et al., 2009), é requerida a instalação de sensores de alto custo. $\mathrm{O}$ sincronismo desses sistemas, por conta da sua complexidade, apresenta desempenho insuficiente, chegando a 50\% dos processos acionados manualmente devido a esse fato (ARRUDA et al., 2008). Nos processos industriais, o ambiente tem uma dimensão especialmente pequena, se comparada à área de uma propriedade agrícola. Assim, a adoção de um grande número de sensores em projetos de produção agrícola pode representar um complicador na operação da planta, devido à distância física. Usar variáveis e dispositivos de controle que diminuam o uso de sensores pode facilitar o projeto e a operação de sistemas de irrigação.

Em sistemas de irrigação automatizados, podem-se usar válvulas de controle hidráulico na seleção de parcelas de irrigação. Nesse caso, quando as válvulas operam, variam a pressão de saída do sistema e a vazão, tornando necessário o ajuste de vazão do sistema. Válvulas reguladoras de pressão ou o controle da velocidade do motor podem ser usados para regular a vazão. As válvulas reguladoras de pressão atuam por meio da variação automática da saída de água da bomba, neste caso, há introdução de perda de carga na curva do sistema, resultando em perda de energia ao sistema (CARVALHO et al., 2000). Usando um inversor de frequência para o ajuste da vazão por intermédio do controle da velocidade do motor, aumenta-se a eficiência energética do sistema, pois a energia que era consumida na perda de carga, devido às válvulas reguladoras, não são mais utilizadas.

Diante das premissas abordadas, buscouse investigar as variáveis do bombeamento de um sistema de irrigação através de analise multivariada. Dessa forma, foi possível identificar a variável que se adequasse a uma estratégia de pseudo controle, dispensando a utilização de sensores de vazão e pressão, o que resulta em um sistema de controle mais simples e de menor custo.

\section{MATERIAL E MÉTODOS}

Partindo-se da hipótese de que a vazão de um sistema hidráulico pode ser controlada automaticamente por meio da rotação do motor, instalou-se um inversor para acionar e monitorar um sistema de moto bomba empregado na irrigação. Para testar a hipótese, montou-se uma bancada de ensaios (Figura 1) a fim de obter o comportamento dos parâmetros (corrente elétrica e rotações por minuto) do motor de indução trifásico (MIT) e da bomba hidráulica (vazão, pressão do recalque e pressão de sucção) em diferentes frequências, usando o inversor para investigar o comportamento da pressão imposta ao sistema.

Esses ensaios foram realizados no laboratório de hidráulica do DENA (Departamento de Engenharia Agrícola), usando uma moto bomba com um motor elétrico de $3 \mathrm{cv}$, um inversor com entrada de 380-480V trifásico, $50 / 60 \mathrm{~Hz}, 8,5 \mathrm{~A}$, saída trifásica de 0 $480 \mathrm{~V}, 8,2 \mathrm{~A} 6,2 \mathrm{KVA} 5 \mathrm{HP}$ e frequência de $0,1-400 \mathrm{~Hz}$ para acionar o motor e controlar a frequência. A medição da corrente elétrica é feita pelo inversor. Uma calha parshall foi montada para a medição da vazão do sistema. Foram realizadas as leituras com seis diferentes frequências $(40 ; 50 ; 52,5 ; 55 ; 57,5 ; 60) \mathrm{Hz}$, cada uma com quatro repetições e as leituras da pressão de recalque realizadas no manômetro de bourdon. Um registro de gaveta foi utilizado para fazer a variação da vazão, simulando o procedimento de campo. Os intervalos diferenciados de frequência de saída do inversor utilizado foram decorrentes de duas razões: a primeira é devido ao fato de que a resposta de variação da vazão ser alta no intervalo de 50 a $60 \mathrm{~Hz}$, enquanto que nas frequencias de 40 e 50 . Em Hz a vazão apresenta menor sensibilidade às flutuações da frequência; a segunda razão que justificou adoção de valores menores ou iguais a $60 \mathrm{~Hz}$, está ligada à construção mecânica do motor. Um motor com velocidades acima da nominal ocasionará desgaste prematuro de suas partes móveis, uma frequência maior que $60 \mathrm{~Hz}$, implica uma rotação maior que a nominal do motor.

Os dados coletados foram processados pelo programa estatístico SPSS v.16.0. A similaridade entre as variáveis foram encontradas utilizando a analise de agrupamento hierárquico (AAH), aplicando o algoritmo "método de Ward" e a similaridade estimada pela Distância Euclidiana Quadrada. Dessa maneira, podemse encontrar os grupos de variáveis que apresentam semelhança entre si, em relação à magnitude dos valores das variáveis estudadas e, assim, estimar parâmetros de interesse para o controle da vazão que apresentam similaridade com essa variável. CARGNELUTTI FILHO et al. (2009) utilizaram o método de Ward para agrupar um conjunto de dados de 


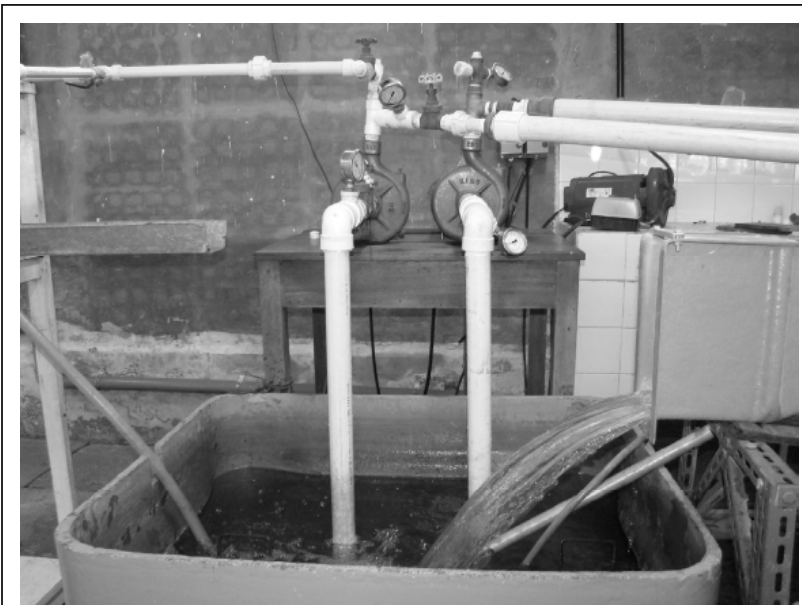

Figura 1 - Bancada de ensaios no laboratório.

variáveis meteorológicas com base temporal e espacial em outra plataforma de processamento.

Para estabelecer a correlação entre as variáveis e identificar os fatores que explicam a variabilidade do sistema, a análise fatorial/análise da componente principal (AF/ACP) foi a técnica utilizada. WEIRICH NETO et al. (2006) relatam que o primeiro fator da análise fatorial considera as correlações mais fortes entre as variáveis com valores de coeficiente de correlação elevados, variáveis independentes apresentam variabilidades semelhantes e podem ser utilizadas para análise e estudo da variável principal.

A avaliação da explicabilidade da correlação foi obtida pro meio da aplicação do teste de Kaiser-Meyer-Olkin (KMO), que verifica o grau de correlação parcial entre os fatores. O Bartlett's teste of sphericity é o que indica se há inadequação dos dados por meio da observação da correlação zero entre as variáveis e o Measure of Sampling Adequacy (MAS) indica essa inadequação por intermédio do exame da diagonal da matriz anti-imagem. A observação da matriz anti-imagem mostra a correlação parcial, sendo um indicador da relação entre duas variáveis, retirando a ligação entre as demais variáveis do sistema. A observação dessa característica pode ser um bom indicador do parâmetro de interesse no controle da vazão.

\section{RESULTADOS E DISCUSSÕES}

A tabela 1 é a coleta de dados quando o inversor de frequência foi configurado para trabalhar

Tabela 1 - Dados coletados no ensaio experimental realizado no laboratório.

\begin{tabular}{|c|c|c|c|c|c|c|c|}
\hline \multirow{2}{*}{\multicolumn{2}{|c|}{ Frequência (Hz): 55}} & \multirow{3}{*}{ H } & \multirow{3}{*}{$\begin{array}{c}\text { Corrente } \\
-\end{array}$} & \multirow{3}{*}{$\frac{\text { Rotação }}{-}$} & \multirow{2}{*}{\multicolumn{2}{|c|}{--------------Vazão--------------- }} & \multirow{3}{*}{$\begin{array}{c}\text { Voltas no Registro } \\
-\end{array}$} \\
\hline & & & & & & & \\
\hline Recalque & Sucção & & & & Altura & Vazão & \\
\hline (mca) & (mca) & $(\mathrm{m})$ & (A) & (RPM) & $(\mathrm{cm})$ & $\left(\mathrm{L} \mathrm{s}^{-1}\right)$ & - \\
\hline 7 & $-4,6$ & 11,6 & 5,1 & 3204 & 17,1 & 3,96133 & 4,25 \\
\hline 7 & $-4,6$ & 11,6 & 5,1 & 3210 & 17,1 & 3,96133 & 4,25 \\
\hline 7 & $-4,8$ & 11,8 & 5,2 & 3210 & 17,5 & 4,12763 & 5 \\
\hline 7 & $-4,8$ & 11,8 & 5,2 & 3209 & 17,5 & 4,12763 & 5 \\
\hline 10 & $-4,5$ & 14,5 & 5,2 & 3210 & 16,9 & 3,8793 & 3,5 \\
\hline 10 & -4 & 14 & 5,1 & 3216 & 16,6 & 3,75766 & 3,25 \\
\hline 10 & $-4,4$ & 14,4 & 5,2 & 3206 & 16,6 & 3,75766 & 3,75 \\
\hline 10 & -4 & 14 & 5,2 & 3211 & 17 & 3,92022 & 3,5 \\
\hline 15 & $-3,5$ & 18,5 & 5,1 & 3217 & 16,2 & 3,59813 & 2,75 \\
\hline 15 & $-3,2$ & 18,2 & 5 & 3222 & 15,4 & 3,2882 & 2,5 \\
\hline 15 & $-3,6$ & 18,6 & 5,1 & 3214 & 16,3 & 3,63773 & 3,25 \\
\hline 15 & $-3,6$ & 18,6 & 5,1 & 3211 & 15,8 & 3,44164 & 2,75 \\
\hline 20 & -2 & 22 & 4,8 & 3239 & 13 & 2,43273 & 2 \\
\hline 20 & $-2,2$ & 22,2 & 4,8 & 3226 & 13,8 & 2,70535 & 1,75 \\
\hline 20 & -2 & 22 & 4,8 & 3224 & 13,3 & 2,53347 & 2 \\
\hline 20 & $-2,2$ & 22,2 & 4,8 & 3226 & 14,3 & 2,88214 & 1,75 \\
\hline 25 & $-0,2$ & 25,2 & 3,9 & 3264 & 0 & 0 & 0 \\
\hline 25 & $-0,2$ & 25,2 & 3,9 & 3227 & 0 & 0 & 0 \\
\hline 25 & $-0,2$ & 25,2 & 4 & 3255 & 0 & 0 & 0 \\
\hline 25 & 0 & 25 & 4 & 3255 & 0 & 0 & 0 \\
\hline
\end{tabular}


com uma frequência de saída de $55 \mathrm{~Hz}$. Observando a pressão de suç̧ão em relação à pressão de recalque, identifica-se que a vazão apresenta uma maior sensibilidade com a pressão de sucção. Tal fato já pode ser um indicativo da melhor adequação do monitoramento da pressão de suç̧ão para o controle de vazão.

Análise de agrupamento hierárquico (AAH)

Quantificar o quanto são parecidos os parâmetros obtidos no ensaio foi o objetivo da $\mathrm{AAH}$ aplicada neste trabalho. Como a distância euclidiana representa uma dissimilaridade, ou seja, quanto maior forem os valores observados entre as variáveis, menos similares (parecidos) serão os objetos, pode-se observar na tabela 2 que a Vazão $\left(\mathrm{L} \mathrm{s}^{-1}\right)$ tem maior semelhança com a Vazão $(\mathrm{cm})$, que, na prática, é um valor de nível proporcional à Vazão do sistema. $\mathrm{O}$ valor da corrente elétrica vem como o terceiro menor valor em relação à Vazão $\left(\mathrm{L} \mathrm{s}^{-1}\right)$, estabelecendo uma forte identidade com essa variável.
Uma melhor visualização desse resultado pode ser vista na figura 2, que identifica as variáveis similares através de um dendrograma. As variáveis que apresentam maior similaridade com a vazão $\left(\mathrm{L} \mathrm{s}^{-1}\right)$ são o nível da calha parshall (vazão $(\mathrm{cm})$ ), voltas do registro e corrente elétrica do motor de indução trifásico.

Análise da Fatorial/Análise da Componente Principal (AF/ACP)

Analisando-se a matriz de correlação das variáveis investigadas (Tabela 3), identifica-se que a altura manométrica total $(\mathrm{Hm})$ foi excluída em decorrência do teste KMO não ser executado se esta se fizesse presente na matriz. Como o teste de KMO expressa a correlação parcial de uma variável em relação às demais, acredita-se que essa correlação seja explicada com uma outra variável que não se faz presente na matriz, portanto ela foi excluída. O resultado do processamento, mostrado na tabela 3 , confirma a alta correlação que existe entre a corrente elétrica do motor

Tabela 2 - Matriz de saída do SPSS mostrando o grau de semelhança entre as variáveis.

\begin{tabular}{|c|c|c|c|c|c|c|c|c|c|}
\hline & $\begin{array}{l}\text { Pressão } \\
\text { recalque } \\
\text { (mca) }\end{array}$ & $\begin{array}{l}\text { Pressão } \\
\text { sucção } \\
\text { (mca) }\end{array}$ & $\begin{array}{l}\mathrm{H} \\
(\mathrm{m})\end{array}$ & $\begin{array}{l}\text { Corrente } \\
\text { (A) }\end{array}$ & $\begin{array}{c}\text { Rot. } \\
\text { (RPM) }\end{array}$ & $\begin{array}{c}\text { Vazão } \\
\text { altura } \\
(\mathrm{cm})\end{array}$ & $\begin{array}{l}\text { Vazão } \\
\left(\mathrm{L} \mathrm{s}^{-1}\right)\end{array}$ & $\begin{array}{c}\text { Voltas no } \\
\text { registro } \\
-\end{array}$ & $\begin{array}{l}\text { Freq. } \\
(\mathrm{Hz})\end{array}$ \\
\hline $\begin{array}{l}\text { Pressão } \\
\text { recalque } \\
\text { (mca) }\end{array}$ & 0 & 85 & 5,3 & 394,3 & 155,6 & 396,6 & 408,7 & 448,7 & 153,2 \\
\hline $\begin{array}{l}\text { Pressão } \\
\text { Sucção } \\
\text { (mca) }\end{array}$ & 85 & 0 & 127 & 480,2 & 302 & 483,8 & 498,7 & 494 & 346,8 \\
\hline $\mathrm{H}(\mathrm{m})$ & 5,3 & 126,9 & 0 & 355,3 & 126,9 & 357,1 & 367,1 & 414,7 & 111,9 \\
\hline Corrente (A) & 394,3 & 480,2 & 355 & 0 & 206,2 & 28 & 22,1 & 50,4 & 163,5 \\
\hline $\begin{array}{l}\text { Rotação } \\
\text { (RPM) }\end{array}$ & 155,6 & 302 & 127 & 206,2 & 0 & 216,2 & 209,3 & 241,8 & 53,6 \\
\hline $\begin{array}{l}\text { Vazão } \\
\text { Altura (cm) }\end{array}$ & 396,6 & 483,8 & 357 & 28 & 216,2 & 0 & 4,5 & 26,6 & 175 \\
\hline $\operatorname{Vazão}\left(\mathrm{L}, \mathrm{s}^{-1}\right)$ & 408,7 & 498,7 & 367 & 22,1 & 209,3 & 4,5 & 0 & 17,6 & 164,3 \\
\hline $\begin{array}{l}\text { Voltas no } \\
\text { registro }\end{array}$ & 448,7 & 494 & 414 & 50,4 & 241,8 & 26,6 & 17,6 & 0 & 211 \\
\hline Freq. (Hz) & 153,2 & 346,8 & 111 & 163,5 & 53,6 & 175 & 164,3 & 211 & 0 \\
\hline
\end{tabular}




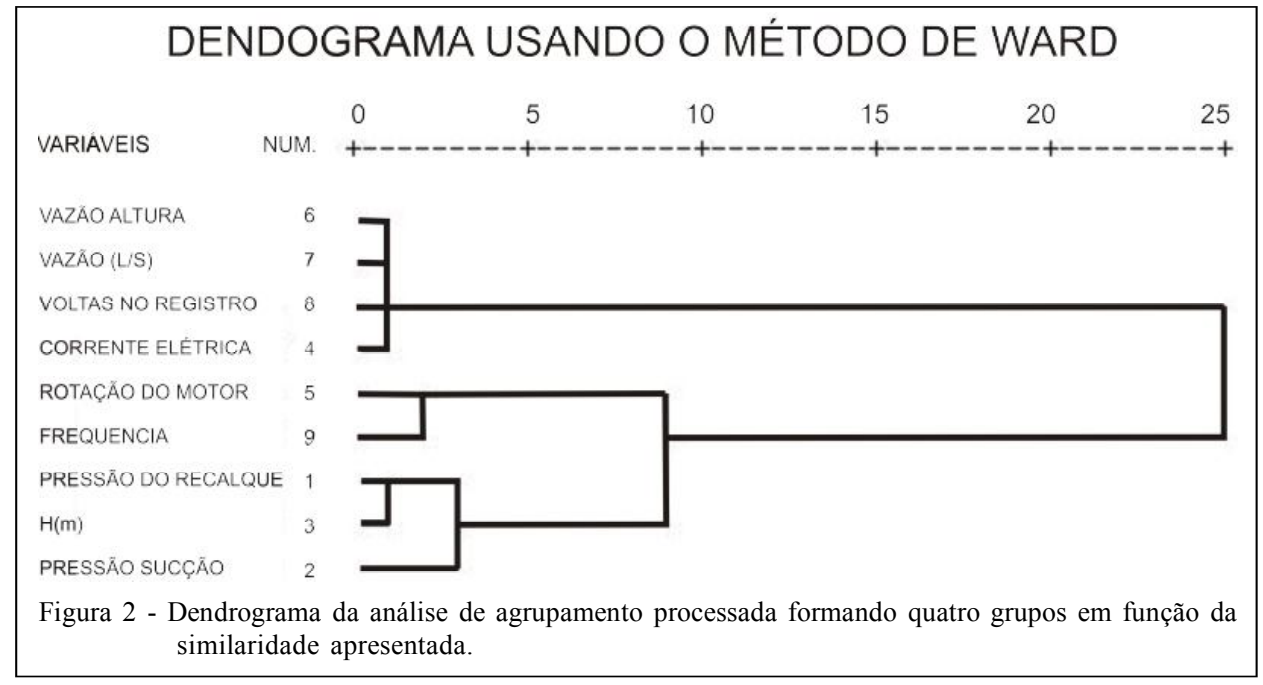

e a vazão, o fator associado a essas duas variáveis é maior que o fator encontrado para pressão de recalque em relação à vazão, estabelecendo uma pseudo variável para o controle da vazão. Segundo ANDRIGHETTO et al. (2008), a corrente elétrica é usada no controle de sobrecarga de motores por meio do alívio do fluxo do processo e, nesse caso, são utilizados sensores de corrente. No inversor usado na coleta de dados, a medida da corrente elétrica é feita pelo próprio inversor, dispensando assim o uso de sensores. SILVA JÚNIOR \& CAGNON (2005) relatam que o controle de velocidade de motores por meio de inversor de frequência também pode trazer a elevação do fator de potência, liberação de carga de transformadores e

Tabela 3 - Matriz de correlação das variáveis estudadas a fim de definir a variável ideal para o controle automático do sistema de irrigação usando o inversor de frequência.

\begin{tabular}{|c|c|c|c|c|c|c|c|c|}
\hline & $\begin{array}{c}\text { Pressão } \\
\text { recalque } \\
\text { (mca) }\end{array}$ & $\begin{array}{l}\text { Pressão } \\
\text { sucção } \\
\text { (mca) }\end{array}$ & $\begin{array}{l}\text { Corrente } \\
\text { (A) }\end{array}$ & $\begin{array}{l}\text { Rot. } \\
\text { (RPM) }\end{array}$ & $\begin{array}{c}\text { Vazão altura } \\
(\mathrm{cm})\end{array}$ & $\begin{array}{l}\text { Vazão } \\
\left(\mathrm{L} \mathrm{s}^{-1}\right)\end{array}$ & $\begin{array}{c}\text { Voltas no } \\
\text { registro } \\
-\end{array}$ & $\begin{array}{l}\text { Freq. } \\
(\mathrm{Hz})\end{array}$ \\
\hline $\begin{array}{l}\text { Pressão Recalque } \\
\text { (mca) }\end{array}$ & 1 & 0,66525 & $-0,5522$ & 0,3873 & $-0,562$ & $-0,609$ & $-0,7666$ & 0,397 \\
\hline $\begin{array}{l}\text { Pressão Sucção } \\
\text { (mca) }\end{array}$ & 0,66525 & 1 & $-0,8905$ & $-0,189$ & $-0,905$ & $-0,963$ & $-0,9449$ & $-0,365$ \\
\hline Corrente (A) & $-0,55221$ & $-0,89054$ & 1 & 0,1880 & 0,889 & 0,913 & 0,80166 & 0,356 \\
\hline Rotação (RPM) & 0,38733 & $-0,1891$ & 0,18804 & 1 & 0,149 & 0,176 & 0,04788 & 0,789 \\
\hline $\begin{array}{l}\text { Vazão Altura } \\
(\mathrm{cm})\end{array}$ & $-0,5616$ & $-0,9047$ & 0,88965 & 0,1487 & 1 & 0,982 & 0,89541 & 0,311 \\
\hline Vazão $\left(\mathrm{L} \mathrm{s}^{-1}\right)$ & $-0,6092$ & $-0,9634$ & 0,91294 & 0,1760 & 0,982 & 1 & 0,9307 & 0,353 \\
\hline $\begin{array}{l}\text { Voltas no } \\
\text { registro }\end{array}$ & $-0,7666$ & $-0,9448$ & 0,8017 & 0,0479 & 0,895 & 0,931 & 1 & 0,169 \\
\hline Freq. (Hz) & 0,39678 & $-0,3653$ & 0,35611 & 0,7888 & 0,311 & 0,353 & 0,16913 & 1 \\
\hline
\end{tabular}


redução do consumo de energia elétrica. A saída de pressão do sistema vai necessitar de um sensor de pressão para fazer o controle automático. Sua instalação, em muitos casos, deve ser em pontos distantes da unidade de controle, o que ocasiona problema de sincronismo e aumento dos custos. Esse foi o parâmetro que apresentou menor correlação com a vazão entre os cincos melhores.

Os testes de explicabilidade e adequacidade aplicado ao modelo, Kaiser-Meyer-Olkin (KMO) e Bartlett's Test of Sphericity, apresentaram um índice igual a 0,747 (bom) e um valor de significância de 0,00, respectivamente, demonstrando que o modelo se ajusta à matriz dos dados originais. Os fatores não explicariam as variações dos parâmetros caso esse coeficiente fosse menor que 0,5 e o teste de significância maior que 0,05 .

As informações das MAS (Measure of Sampling Adequacy), presentes na tabela 4, expressam que todas as variáveis estudadas contribuem significativamente $(\mathrm{P} \quad 0,05)$ para a explicabilidade da variância dos dados originais, sendo o menor valor igual a 0,52. O modelo que melhor se ajustou aos dados foi aquele composto por duas componentes com as raízes características superiores à unidade e explicando $91 \%$ da variância total (Tabela 4). As duas componentes expressam a relação entre fatores e variáveis e permite a identificação das variáveis com maiores inter-relações em cada componente. Os valores elevados dos pesos fatoriais sugerem quais são as variáveis mais significativas em cada fator. No primeiro fator, cinco variáveis apresentaram pesos superiores a 0,93 , indicando as variáveis mais significativas que podem ser monitoradas para se fazer o controle da vazão do sistema por intermédio de uma estratégia que utilize uma dessas variáveis. Essa estratégia usará o parâmetro que ocasionará o menor custo e a menor complexidade ao sistema de controle.

\section{CONCLUSÃO}

O controle da vazão pode ser feito monitorando sua variação na saída do sistema, porém há necessidade de sensores de alto custo e a distância do sensor até a unidade de controle pode trazer problemas de sincronismo e custo ao sistema, devido à instalação.

A sucção está próxima do sistema de controle, mas precisa de um sensor para se estabelecer a malha de controle, no entanto foi o parâmetro que apresentou melhor correlação com a vazão. Esse fato nos leva a concluir que o monitoramento da pressão de sucção é mais interessante que o monitoramento da pressão de saída para controle de vazão dos sistemas de irrigação.

O posicionamento do registro também pode ser usado, mas a principal dificuldade vai ser a distância ao sistema de controle e o uso dessa variável para o controle pode precisar de um sistema de sensores simples.

A corrente elétrica é medida pelo o inversor, logo já está sendo medida no sistema de controle e acionamento do motor. Assim, é conveniente utilizar o monitoramento da corrente para controle automático da vazão do sistema de irrigação, pois não há necessidade de sensor remoto.

Tabela 4 - Agrupamento dos fatores pela componente principal.

\begin{tabular}{|c|c|c|c|}
\hline \multirow{3}{*}{ Variáveis } & . & ----------" & \multirow{3}{*}{ MAS (Medida de adequação da amostra) } \\
\hline & & & \\
\hline & 1 & 2 & \\
\hline Vazão $\left(\mathrm{L} \mathrm{s}^{-1}\right)$ & 0,990 & - & 0,676 \\
\hline Pressão sucção & $-0,982$ & - & 0,765 \\
\hline Vazão altura $(\mathrm{cm})$ & 0,958 & - & 0,889 \\
\hline Voltas no registro & 0,955 & $-0,158$ & 0,772 \\
\hline Corrente elétrica & 0,927 & - & 0,699 \\
\hline Pressão Recalque & $-0,674$ & 0,665 & 0,736 \\
\hline Frequencia & 0,323 & 0,907 & 0,842 \\
\hline Rotação & 0,170 & 0,899 & 0,520 \\
\hline Autovalor & 5,221 & 2,106 & \\
\hline \% variância explicada & 65,266 & 26,319 & \\
\hline \% variância acumulada & 65,266 & 91,585 & \\
\hline
\end{tabular}

Ciência Rural, v.40, n.11, nov, 2010. 


\section{REFERÊNCIAS}

ANDRIGHETTO, P.L. et al. Controle automático de registros de descarga com acionamento pneumático em unidades de armazenagem agrícola. Ciência Rural, Santa Maria, v.38, n.9, p.2626-2629, 2008. Disponível em: $<$ http://www.scielo.br/scielo.php?script $=$ sci_arttext\&pid=S0103-84782008000900036\&lng= en\&nrm=iso $>$. Acesso em: 26 maio, 2010. doi: 10.1590/S010384782008005000017 .

ARRUDA, L.V.R. de et al. Um método evolucionário para sintonia de controladores PI/PID em processos multivariáveis. Sba Controle \& Automação, Natal, v.19, n.1, p.1-17, 2008. Disponível em: $<$ http://www.scielo.br/scielo.php?script $=$ sci_arttext\&pid $=$ S010317592008000100001\&lng=pt\&nrm=iso $>$. Acesso em: 27 maio, 2010. doi: 10.1590/S0103-17592008000100001.

CARGNELUTTI FILHO, A. et al. Variabilidade temporal e espacial da precisão das estimativas de elementos meteorológicos no Rio Grande do Sul. Ciencia Rural, Santa Maria, v.39, n.4, jul. 2009. Disponível em: $<$ http://www.scielo.br/scielo.php?script=sci_arttext\&pid=S0103$84782009000400002 \& \operatorname{lng}=\mathrm{en} \& \mathrm{nrm}=\mathrm{iso}>$. Accesso em: 27 maio, 2010. doi: 10.1590/S0103-84782009005000051.

CARVALHO, J.A. et al. Utilização do inversor de frequencia em sistemas de irrigação para controle de vazão. Revista Brasileira de Engenharia Agrícola e Ambiental, Campina Grande local, v.4, n.1, p.51-56, 2000.

CORRAR, L.J. et al. Analise multivariada: para os cursos de administração, ciências contábeis e economia. São Paulo: Atlas, 2007. 541p.
OGATA, K. Engenharia de controle moderno. 4.ed. São Paulo: Pearson Prentice Hall, 2003. 788p.

SILVA JÚNIOR, A.N.; CAGNON, J.A. Armazenagem de grãos: Economia de energia elétrica possibilitada pelo uso do inversor de freqüência. Energenharia Agricola, Botucatu, v.20, n.3, p.3149, 2005. Disponível em: <http://link.periodicos.capes.gov.br/ sfxlc13?url_ver=Z39.88-2004\&url_ctx_fmt $=$ infofi/ fmt:kev:mtx:ctx\&ctx_enc=info:ofi/enc:UTF-8\&ctx_ver=Z39.882004\&rfr_id=info:sid/sfxit.com:azlist\&sfx.ignore_date_th reshold=1\&rft.object_id=991042752512342>. Acesso em: 25 de maio, 2010.

WAHAB, N.A. et al. Multivariable PID control design for activated for sludge process with nitrification and denitrification. Biochemical Engineering Journal, Manchester - EUA, n.45, p.239-248, 2009. Disponível em: <http://www.sciencedirect.com/ s c i e n c e/artic 1 e/ B 6 V 5 N-4 W 6 Y 39 C-1/2/ 6be605422fa514bc35944bab09f3932a>. Acesso em: 27 maio, 2010. doi: 10.1016/j.bej.2009.04.016.

WEIRICH NETO, P.H. et al. Análise multivariada da resistência do solo à penetração sob plantio direto. Ciencia Rural, Santa Maria, v.36, n.4, p.1186-1192, 2006. Disponível em: <http:/ /www.scielo.br/scielo.php?script $=$ sci_arttext\&pid $=$ S0 103 $84782006000400022 \& \operatorname{lng}=\mathrm{en} \& n \mathrm{~nm}=\mathrm{iso}>$. Accesso: 26 maio, 2010. doi: 10.1590/S0103-84782006000400022. 